\title{
Role of perineural invasion as a prognostic factor in laryngeal cancer
}

\author{
MASSIMO MESOLELLA ${ }^{1}$, BRIGIDA IORIO ${ }^{1}$, GABRIELLA MISSO ${ }^{2}$, AMALIA LUCE ${ }^{2}$, \\ MARIANO CIMMINO ${ }^{1}$, MAURIZIO IENGO ${ }^{1}$, MARIO LANDI ${ }^{1}$, PASQUALE SPERLONGANO ${ }^{3}$, \\ MICHELE CARAGLIA $^{2}$ and FILIPPO RICCIARDIELLO ${ }^{1}$
}

\author{
${ }^{1}$ Department of Neurosciences, Ear, Nose and Throat Section, University of Naples 'Federico II'; \\ ${ }^{2}$ Department of Biochemistry, Biophysics and General Pathology, Second University of Naples, Naples I-80138; \\ ${ }^{3}$ Unit of General and Geriatric Surgery, School of Medicine, Second University of Naples, Naples I-80137, Italy
}

Received March 12, 2015; Accepted August 21, 2015

DOI: $10.3892 / \mathrm{ol} .2016 .4265$

\begin{abstract}
The diffusion of laryngeal cancer cells in the perineural space is a parameter associated with a negative prognosis, high loco-regional recurrence and low disease-free survival rates. The spread of tumor cells on the perineural sheath highlights the histopathological and clinically aggressive behavior of this type of tumor, which may extend proximally or distally in the nerve for $>10 \mathrm{~cm}$. Therefore, the surgical resection margin is generally insufficient to treat patients with laryngeal cancer presenting with perineural invasion (PNI) with surgery alone. In PNI, the minor laryngeal nerves are frequently involved, rather than the superior and inferior laryngeal nerves. The aim of the present study was: i) To evaluate the prognostic importance of PNI; ii) to correlate the rate of infiltration with factors associated with the tumor, including histotype, site and tumor-node-metastasis stage, and with the type of surgery (total or partial laryngectomy); and iii) to evaluate the rate of disease-free survival according to the outcome of combined surgery and radiotherapy (RT) treatment, by means of retrospective analysis. The results of the present study highlighted the importance of performing a closer clinical and instrumental follow-up in patients with laryngeal cancer whose histopathological examination is positive for PNI. In such cases, it is important to complement the surgical therapeutic treatment with adjuvant RT.
\end{abstract}

\section{Introduction}

Perineural invasion (PNI), also known as neurotropic carcinomatous spread, is a process predominantly characterized by

Correspondence to: Professor Michele Caraglia, Department of Biochemistry, Biophysics and General Pathology, Second University of Naples, Via Luigi De Crecchio 7, Naples I-80138, Italy

E-mail: michele.caraglia@alice.it

Key words: perineural invasion, laryngeal cancer, prognostic factor, lymph node metastasis, radiotherapy neoplastic invasion of the nerves. PNI may also occur in the absence of lymphatic or vascular invasion (1-5).

In PNI, the propagation of neoplastic cells to the primitive site of the tumor does not occur via lymphatic dissemination, but through molecular mediators that guide these cells during the invasion of the nerve sheath (6). The definition of PNI was first proposed in a study by Batsakis (3), according to which, PNI was 'a tumor cell invasion in, around and through the nerve'. Following multiple modifications, PNI is currently defined as 'the presence of tumor cells in the 3 layers of the nerve sheath' or 'in close proximity to nerve, involving at least $33 \%$ of its entire circumference'; below this cut-off, focal abutment rather than PNI should be considered $(1,6)$.

The incidence of PNI has been reported to vary from 6 to $50 \%$ for head and neck cancer, and $<80 \%$ for squamocellular carcinoma. PNI is an independent negative prognostic factor associated with a lesser loco-regional control of the disease and poor survival in patients with laryngeal cancer (1,2,6-14). The aim of the present study was: i) To evaluate the prognostic importance and incidence of PNI; ii) to correlate the infiltration rate with factors associated to the tumor, including histotype, site and tumor-node-metastasis stage (TNM), and with the type of surgery (total or partial laryngectomy); iii) to evaluate the disease-free survival rate according to the outcome of combined surgery and radiotherapy (RT) treatment; and iv) to formulate a specific follow-up protocol for patients with laryngeal cancer and PNI, depending on the clinical and histopathological characteristics of the tumor.

\section{Patients and methods}

Patients and treatment. The cohort in the present study consisted of 40 patients, 33 of which were male and 7 of which were female. The patients were selected by retrospective analysis of 253 patients who had undergone surgical laryngectomy for laryngeal neoplasia at the Department of Neurosciences, Ear, Nose and Throat (ENT) Section, University of Naples 'Federico II' (Naples, Italy) between 2000 and 2011. The included patients $(n=40)$ were selected from the total records $(n=253)$ based on the following management inclusion criteria: i) Adjuvant RT treatment (60-66 Gy; 2 Gy/day over 6.0-6.5 weeks); ii) oncological, 
Table I. Pathological tumor-node-metastasis analysis of patients with laryngeal cancer and perineural invasion, according to the Union for International Cancer Control and American Joint Committee on Cancer staging system.

\begin{tabular}{|c|c|c|c|c|c|c|c|c|c|}
\hline \multirow[b]{2}{*}{ Patients } & \multicolumn{3}{|c|}{ Total laryngectomy } & \multicolumn{3}{|c|}{ Supraglottic laryngectomy } & \multicolumn{3}{|c|}{ Supracricoid laryngectomy } \\
\hline & T3 & $\mathrm{T} 4$ & Total & T3 & $\mathrm{T} 4$ & Total & $\mathrm{T} 3$ & $\mathrm{~T} 4$ & Total \\
\hline N0 & 9 & 14 & 23 & 1 & 3 & 4 & - & 1 & 1 \\
\hline N1 & 1 & 3 & 4 & 1 & - & 1 & - & 1 & 1 \\
\hline $\mathrm{N} 2$ & 1 & 3 & 4 & - & 1 & 1 & - & 1 & 1 \\
\hline N3 & - & - & - & - & - & - & - & - & - \\
\hline Total & 11 & 20 & 31 & 2 & 4 & 6 & - & 3 & 3 \\
\hline
\end{tabular}

Table II. Mortalities during follow-up.

\begin{tabular}{lccc}
\hline Cause of mortality & No. of patients & Pathological tumor-node-metastasis status, $\mathrm{n}$ & Surgical procedure \\
\hline Laryngeal cancer & 11 & 3 T4N0, 1 T4N1, 3 T4N2, 2 T3N0, 1 T3N1, 1 T3N2 & 7 TL, 3 SGL, 1 SCL \\
Other causes & 2 & 1 T4N0, 1 T3N0 & 2 TL \\
\hline
\end{tabular}

TL, total laryngectomy; SGL, supraglottic laryngectomy; SCL, supracricoid laryngectomy.

clinical and instrumental follow-up through visits and laryngoscopy with optical fibers in the dedicated Ambulatory Centre, conducted monthly in the first year, every 3 months in the second year, and biannually from the third year; and iii) annual positron emission tomography-computed tomography (PET-CT) for locoregional and distant control of the disease. Patients with follow-up times of $<12$ months were excluded from the study. Of the 40 patients included in the study, 31 had been subjected to total laryngectomy (TL), 6 to supraglottic laryngectomy (SGL) and 3 to supracricoid laryngectomy (SCL). All patients were diagnosed with PNI, according to histological and immunohistochemical examination, which revealed the presence of tumor cells invading the 3 nerve sheath layers or involving $\leq 33 \%$ of the entire circumference of the nerve. Written informed consent was obtained from all patients for inclusion in the present study. The study was approved by the ethics committee of ENT Section, University of Naples 'Federico II'.

Statistical analysis. Statistical analysis was performed with MedCalc software, version 9.3.7.0 (MedCalc Software bvba, Ostend, West Flanders, Belgium) by scanning the quantitative variables with the standard functions. The disease-free survival rate was estimated with a Kaplan-Meier curve. $\mathrm{P}<0.05$ was considered to indicate a statistically significant difference.

\section{Results}

Clinical and pathological characteristics. Of the 40 patients examined: i) 31 had undergone TL, 25 of which presented with neoformations that initially involved the vocal folds (10 T4N0, 7 T3N0, 3 T4N1, 3 T4N2, 1 T3N1 and 1 T3N2). In 4 cases, the neoplasia involved the supraglottic region ( $3 \mathrm{~T} 4 \mathrm{~N} 0$ and $1 \mathrm{~T} 3 \mathrm{~N} 0$ ), and in 2 cases the presence of a glottis tumor with subglottic extension was observed (1 T3N0 and 1 T4N0). ii) 6 patients whose tumor involved the supraglottic area had undergone SGL
(3 T4N0, 1 T3N0, 1 T3N1 and 1 T4N2). iii) 3 patients had undergone subtotal reconstructive laryngectomy (SRL), 2 of which presented with supraglottic (1 T4N0 and 1 T4N1) and 1 with glottic (1 T4N2) localization of the tumor.

The pathological tumor-node-metastasis (pTNM) analysis (Table I), was performed according to the criteria described in the 7th edition of the Union for International Cancer Control and American Joint Committee on Cancer (AJCC) staging system, published in 2010 (15), which classified 12 cases with disease stage III and 28 cases with disease stage IV. Microscopic analysis of the tumor specimens revealed the squamocellular histotype in 39 patients $(97.5 \%$; 29 G3 and 10 G2-G3), 12 of which exhibited basaloid characteristics, and only 1 patient with sarcomatoid histology (1 G3).

Survival. During the follow-up, 13 patients succumbed (mean time, 24 months post-treatment); 11 due to laryngeal cancer (3 T4NO, 3 T4N2, 2 T3N0, 1 T3N1, 1 T4N1 and 1 T3N2; 3 in stage III and 8 in stage IV), and 2 due to other causes ( 1 T4N0 and 1 T3N0). The 11 patients who succumbed to the disease presented with locoregional recurrences of laryngeal cancer and/or laterocervical metastases that led to mortality a few months later. In addition, distant metastases were also noted in 2 cases. Among these 11 mortalities, 7 patients had undergone TL, 3 SGL and 1 SRL. Furthermore, histological analysis revealed basaloid aspects of the tumor in 3 cases ( 2 T4N2 and $1 \mathrm{~T} 3 \mathrm{~N} 0$ ). Of the remaining 27 patients, 22 underwent TL, 3 SGL and 2 SRL. Additionally, 19 of these patients were in stage IV and 8 in stage III. These patients continued the clinical and instrumental oncological follow-up, and no clinical or radiological locoregional recurrence has been observed to date (Table II).

Statistical analysis. The statistical analysis indicated that the median survival time was 36 months for the patients in stage III 
and 30 months for those patients in stage IV and this difference was found to be statistically significant $(\mathrm{P}<0.05)$. Following 18 months of treatment, the disease-free survival rate was $100 \%$, with an average follow-up time of 42.35 months.

\section{Discussion}

PNI represents an independent negative prognostic factor associated with increased locoregional recurrence and metastatic laterocervical lymphadenopathies, which adversely affect the prognosis of patients with larynx cancer, in terms of overall and disease-free survival rates (1,2,6-8,10-14). In the 7 th edition of the AJCC staging system for the classification of squamous cell carcinomas, published in 2010, PNI was included among the high-risk factors for patients with laryngeal cancer (16). Previous studies by Soo et al (8) reported that the survival rate of patients with laryngeal cancer were reduced from 49 to $23 \%$ following 3 years of treatment if the patients developed PNI.

In the past, the pathogenic model of laryngeal cancer with PNI was considered to be associated with the lymphatic dissemination of tumor cells within the nerve (8). However, this model was later rejected, since the lymphatic channels do not penetrate the inner sanctum of the nerve sheath. Instead, it has now been proposed that tumor cells spread along neural sheaths in low-resistance planes, using the chemotactic effect of certain neurotrophic molecules, including nerve growth factor, brain-derived neurotrophic factor and neurotrophin-3, which guide the dissemination and invasion of these nerve cells through specific receptor sites (6).

Magnetic resonance imaging (MRI) with contrast has been suggested to be the best imaging method to detect PNI $(11,17)$. Previous studies have demonstrated the preeminence of MRI high-field 3.0-T over traditional MRI 1.5 in spatial resolution, due to the higher signal intensity and the slight difference on contrastographic enhancement of nerves stumps (18-20). Therefore, a refinement of this radiological technique may offer a more sensitive pre-surgical determination of PNI, which nowadays requires the histological analysis of surgical samples (19).

The MRI analysis performed on 13 patients in the present study did not provide any additional information regarding PNI, compared with CT and PET-CT. Tumor cells invading the nerve sheaths may extend proximally or distally in the nerve overtaking the resection margins, which may overturn the significance of surgical resection, since tumor cells can spread anywhere between 2 and $12 \mathrm{~cm}$ from the tumor primitive site without invading the intermediate nerve segments (7).

In previous studies, PNI exhibited a preference for growth along the nerves that infiltrated the intracranial fossa, in particular cranial nerve V. PNI may be incidental without symptoms, or may present clinically with lesions in the cranial nerves. The initial symptoms of PNI include paresthesia and formication, but may slowly progress to pain, numbness and facial weakness (termed Bell's palsy or trigeminal neuralgia) (16).

PNI affects a small percentage of the 'major' nerves, including the laryngeal, hypoglossal, spinal and cervical plexuses ( 2 vs. $33.5 \%$ of the so-called 'minor' nerves with a diameter of $<1 \mathrm{~mm})(11,13)$. Fagan et al (1) reported that nerves with a diameter of $<0.2 \mathrm{~mm}$ were involved in $60 \%$ of PNI cases.

The College of American Pathologists (14) reported that the association between PNI and patient prognosis does not depend on the diameter of the nerves involved. This complicates the pre-surgical identification of PNI through imaging and the histological analyses of these nerves. A number of studies have previously suggested that the appearance of distant lymph node metastases in patients with laryngeal cancer is due to the presence of PNI, which is usually associated with worse locoregional control of the disease (14). Considering the impact on prognosis, the presence of PNI affects the therapeutic options and the decision making process in patients with laryngeal cancer. In this regard, adjuvant radio-chemotherapy has been demonstrated to result in a better outcome in those patients presenting with PNI, in terms of recurrence, local control of the disease, overall survival and disease-free survival rates $(2,14,16,21)$.

The optimal treatment for patients with resectable disease is possibly resection and post-operative RT, while those patients with unresectable cancer are generally treated with definitive RT $(7,16)$. Tumor cells have the tendency to concentrate in the nerve periphery (known as the perinevrium), which is a hypoxic space due to its poor blood supply. This leads to low radiosensitivity in these areas, which may explain the failure of RT treatment observed in previous cases (11).

Previous studies have confirmed that patients with PNI whose nodes are clinically and instrumentally negative, exhibit an increased rate of spread to the regional nodes $(1,2,16,21-24)$. In consequence, these patients should be subjected to elective neck dissection. Furthermore, since the dominant pattern of recurrence is local, strategies aimed to improve local control of the disease, such as chemotherapy, should be considered $(1,2,16,21-24)$.

Miller et al (24) demonstrated an association between the extent of PNI and the disease-free survival time with a novel classification system of PNI subcategories, namely intratumoral, peripheral or extratumoral PNI, according to the distance in $\mathrm{mm}$ to the tumor edge.

The results of the present study suggested that the presence of PNI affects the prognosis of patients with laryngeal cancer, depending on the type of treatment received, the histotype of the tumor and the pTNM stage. Out of the 11 patients who succumbed to the disease in the present study ( 8 in stage IV and 3 in stage III), 7 cases $(63.6 \%$ ) had undergone TL, 3 SGL and 1 SRL. Of these 11 patients, 8 presented with a squamocellular histotype (7 G3 and 1 G2-G3) and 3 with a basaloid histotype (2 G3 and $1 \mathrm{G} 2-\mathrm{G} 3$ ). In addition, 6 patients presented with lymph node metastases. The prevalence of the disease at advanced stage, the high level of undifferentiated cells and the histopathological features associated with PNI, are suggestive of the high aggressiveness displayed by this type of cancer. The incidence of PNI in the present study was $15.81 \%$. The median survival time of the 27 patients with laryngeal cancer who were subjected to surgery ( 22 TL vs. 5 partial laryngectomy) and continuous follow-up was 34 months, which highlights the requirement for a more aggressive surgical treatment and adjuvant RT in order to achieve higher overall and disease-free survival rates for laryngeal cancer patients affected by PNI. The survival rate observed in the present study was higher than 
that reported in the literature (67.5 vs. $30-40 \%$ for patients with local advanced disease), which supports the therapeutic actions and the distance control strategies adopted in the present study.

In conclusion, the presence of PNI represents a valuable prognostic factor that establishes the low survival rates in patients with laryngeal cancer, and confirms the requirement for combined surgical and RT treatment in such patients. If monitored by a rigorous follow-up, the presence of PNI allows the identification of possible recurrences early, so as to establish appropriate salvage therapeutic protocols.

\section{References}

1. Fagan JJ, Collins B, Barnes L, D'Amico F, Myers EN and Johnson JT: Perineural invasion in squamous cell carcinoma of the head and neck. Arch Otolaryngol Head Neck Surg 124 637-640, 1998.

2. Yilmaz T, Hosal AS, Gedikoğlu G, Onerci M and Gürsel B: Prognostic significance of vascular and perineural invasion in cancer of the larynx. Am J Otolaryngol 19: 83-88, 1998.

3. Batsakis JG: Nerves and neurotropic carcinomas. Ann Otol Rhinol Laryngol 94: 426-427, 1985.

4. Olsson Y: Microenvironment of the peripheral nervous system under normal and pathological conditions. Crit Rev Neurobiol 5: 265-311, 1990.

5. Akert K, Sandri C, Weibel ER, Peper K and Moor H: The fine structure of the perineural endothelium. Cell Tissue Res 165: 281-295, 1976.

6. Liebig C, Ayala G, Wilks JA, Berger DH and Albo D: Perineural invasion in cancer: A review of the literature. Cancer 115: 3379-3391, 2009.

7. Vural E, Fan CY, Spring P, Stack BC Jr, Kokoska MS, Hanna EY and Suen JY: Evaluation of the inferior and superior laryngeal nerve stumps for perineural spread in laryngeal cancer. Otolaryngol Head Neck Surg 137: 889-892, 2007.

8. Soo KC, Carter RL, O'Brien CJ, Barr L, Bliss JM and Shaw HJ: Prognostic implications of perineural spread in squamous carcinomas of the head and neck. Laryngoscope 96: $1145-1148,1986$.

9. Kurtz KA, Hoffman HT, Zimmerman MB and Robinson RA: Perineural and vascular invasion in oral cavity squamous carcinoma: Increased incidence on re-review of slides and by using immunohistochemical enhancement. Arch Pathol Lab Med 129: 354-359, 2005.

10. Bova R, Goh R, Poulson M and Coman WB: Total pharyngolaryngectomy for squamous cell carcinoma of the hypopharynx: A review. Laryngoscope 115: 864-869, 2005

11. Selçukbiricik F, Tural D, Büyükünal E and Serdengeçti S: Perineural invasion independent prognostic factors in patients with gastric cancer undergoing curative resection. Asian Pac J Cancer Prev 13: 3149-3152, 2012.
12. Ch'ng S, Maître A, Lea R, Brash H and Tan ST: Parotid metastasis - an independent prognostic factor for head and neck coetaneous squalors cell carcinoma. J Plast Reconstr Aesthet Surg 59: 1288-1293, 2006.

13. Chirilă M, Bolboacă SD, Cosgarea M, Tomescu E and Mureşan M: Perineural invasion of the major and minor nerves in laryngeal and hypopharyngeal cancer. Otolaryngol Head Neck Surg 140: 65-69, 2009.

14. College of American Pathologists: Protocol for the Examination of Specimens From Patients With Carcinomas of the Pharynx. College of American Pathologists, Northfield, IL, USA, 2007. http://cap.org/apps/docs/committees/cancer/ cancer_protocols/2012/Pharynx_12protocol.pdf. Accessed May 10,2015.

15. Edge SB, Byrd DR, Compton CC, Fritz AG, Greene FL and Trotti A (eds): AJCC Cancer Staging Manual. 7th edition. Springer, New York, 2010.

16. Mendenhall WM, Ferlito A, Takes RP, Bradford CR, Corry J, Fagan JJ, Rinaldo A, Strojan P and Rodrigo JP: Cutaneous head and neck basal and squamous cell carcinomas with perineural invasion. Oral Oncol 48: 918-922, 2012.

17. Galloway TJ, Morris CG, Mancuso AA, Amdur RJ and Mendenhall WM: Impact of radiographic findings on prognosis for skin carcinoma with clinical perineural invasion. Cancer 103: 1254-1257, 2005.

18. Becker $M$ and Zaidi $H$ : Imaging in head and neck squamous cell carcinoma: The potential role of PET/MRI. Br J Radiol 87: 20130677, 2014

19. Penn R, Abemayor E, Nabili V, Bhuta S and Kirsch C: Perineural invasion detected by high-field 3.0-T magnetic resonance imaging. Am J Otolaryngol 31: 482-484, 2010.

20. Fischbach F, Müller M and Bruhn H: Magnetic resonance imaging of the cranial nerves in the posterior fossa: A comparative study of t2-weighted spin-echo sequences at 1.5 and 3.0 tesla. Acta Radiol 49: 358-363, 2008.

21. Kropp L, Balamucki CJ, Morris CG, Kirwan J, Cognetta AB, Stoer CB and Mendenhall WM: Mohs resection and postoperative radiotherapy for head and neck cancers with incidental perineural invasion. Am J Otolaryngol 34: 373-377, 2013.

22. Ozdek A, Sarac S, Akyol MU, Unal OF and Sungur A: Histopathological predictors of occult lymph node metastases in supraglottic squamous cell carcinomas. Eur Arch Otorhinolaryngol 257: 389-392, 2000.

23. Myers EN and Fagan JF: Management of the neck in cancer of the larynx. Ann Otol Rhinol Laryngol 108: 828-832, 1999.

24. Miller ME, Palla B, Chen Q, Elashoff DA, Abemayor E, St John MA and Lai CK: A novel classification system for perineural invasion in noncutaneous head and neck squamous cell carcinoma: Histologic subcategories and patient outcomes. Am J Otolaryngol 33: 212-215, 2012. 